\title{
XPA serves as an autophagy and apoptosis inducer by suppressing hepatocellular carcinoma in a PI3K/Akt/mTOR dependent manner
}

\author{
Yi Deng ${ }^{1,2 \#}$, Qing-Song Chen ${ }^{1,3 \#}$, Wei-Feng Huang ${ }^{1}$, Jiang-Wen Dai ${ }^{1,4}$, Zhong-Jun Wu ${ }^{1 \wedge}$ \\ ${ }^{1}$ Department of Hepatobiliary Surgery, the First Affiliated Hospital of Chongqing Medical University, Chongqing, China; ${ }^{2}$ Department of Oncology, \\ Yongchuan Hospital of Chongqing Medical University, Chongqing, China; ${ }^{3}$ Department of Traumatology, Chongqing University Central Hospital, \\ Chongqing, China; ${ }^{4}$ Department of Oncology, Chengdu Fifth People's Hospital, Chengdu, China \\ Contributions: (I) Conception and design: Y Deng, QS Chen; (II) Administrative support: ZJ Wu; (III) Provision of study materials or patients: \\ QS Chen, WF Huang; (IV) Collection and assembly of data: JW Dai, QS Chen; (V) Data analysis and interpretation: Y Deng, QS Chen; (VI) \\ Manuscript writing: All authors; (VII) Final approval of manuscript: All authors. \\ \#These authors contributed equally to this work and should be regarded as co-first authors. \\ Correspondence to: Zhong-Jun Wu. Department of Hepatobiliary Surgery, the First Affiliated Hospital of Chongqing Medical University, Chongqing, \\ China. Email: wzjtcy@126.com.
}

Background: To explore the potential biological function of XPA (Xeroderma pigmentosum group A) in hepatic neoplasms and the underlying molecular mechanisms.

Methods: Liver cells were used as experimental models to establish HCC (hepatocellular carcinoma) in vitro. Protein extractions were subjected to Western blotting to detect the proteins expression. The lentivirus transfection efficiency was confirmed by Western blot and RT-qPCR, Tunnel staining was used to detect apoptosis, and Transwell assays were used to observe cell migration and invasion. Cell proliferation was detected with colony formation and CCK-8 (cell counting kit-8) assays.

Results: XPA expression was obviously lower in HCC tissue and liver cancer cell lines. XPA overexpression induced autophagy and apoptosis by increasing LC3B II/I, Beclin1, cleaved-caspase-3, and Bax expression and decreasing p62 and Bcl2 protein levels. XPA also suppressed HCC EMT (Epithelial-Mesenchymal Transition) by increasing E-cadherin and decreasing $\mathrm{N}$-cadherin and vimentin protein expression. Cell proliferation, migration and invasion in vivo were significantly inhibited by the overexpression of XPA, and p-PI3K, p-Akt, and p-mTOR expression were decreased in LV-XPA cells. In general, XPA inhibited HCC by inducing autophagy and apoptosis and by modulating the expression of PI3K/Akt/mTOR proteins.

Conclusions: XPA overexpression was found to suppress HCC by inducing autophagy and apoptosis and repressing EMT and proliferation. Each of these effects may be involved in modulating the PI3K/Akt/ mTOR signaling pathway.

Keywords: XPA; autophagy; apoptosis; EMT; PI3K/Akt/mTOR

Submitted May 15, 2021. Accepted for publication Jul 26, 2021.

doi: 10.21037/jgo-21-310

View this article at: https://dx.doi.org/10.21037/jgo-21-310

^ ORCID: Yi Deng, 0000-0003-4577-1645; Zhong-Jun Wu, 0000-0002-1627-0633. 


\section{Introduction}

Hepatocellular carcinoma (HCC) is a major clinical health problems and ranks second in terms of the number of malignant tumor-related deaths worldwide (1). HCC patients currently receive conventional treatment such as chemotherapy, hepatic resection, and Chinese herbal medicine, but the outcomes are unsatisfactory and have not effectively improved the 5 -year survival rate. In addition, $50 \%$ of patients receiving these therapies will suffer from a recurrence within 2 years (2). Therefore, the need for more robust treatments for HCC is strong and identifying specific HCC target mutant genes and exploring their potential molecular mechanisms will strongly facilitate the development of novel strategies for HCC treatment.

Xeroderma pigmentosum group A (XPA), located on chromosome $9 \mathrm{q} 22.3$, is a major subunit of the nucleotide excision repair (NER) system that can effectively recognize DNA damage then recruit NER proteins to the damaged areas $(3,4)$. Many studies have shown that a lack of XPA leads to the complete failure of NER $(5,6)$, and poor XPA expression in colorectal cancer was identified as a novel biomarker as it could predict the outcomes of colorectal cancer patients (7). Multiple genetic variants of DNA repair genes, including XPA rs2808668, underwent genegene interactions in lung cancer, and may be caused by smoking (8). Moreover, XPA variation was associated with an increase in lung cancer morbidity and was considered to be a low-penetrant risk factor (9), and bladder cancer showed decreased XPA expression leading to carcinogenesis by disturbing DNA repair (10). While these and other reports on the role of XPA in carcinomas seem to conclude that XPA may have a DNA repair function, its biological functions and potential molecular mechanism in HCC remain largely unknown and require urgent investigation.

Autophagy is a highly conserved mechanism with four classical stages; initiation, elongation, autophagosome formation, and autophagolysosome formation (11), and the three well-known types are macroautophagy, microautophagy, and chaperone-mediated autophagy (12). Autophagy has been shown to both suppress HCC and promote HCC. Genetic modification of Beclin $1^{-/}$mice led to a higher prevalence of HCC (13), while mice specific to $\operatorname{Atg} 7^{-/-}$ showed a suppressive function of hepatic adenomas (14). The various functions of autophagy are achieved through many autophagy-related genes (Atg) and among these, LC3, Beclin1, and p62 are the most frequently researched in various malignancies. LC3, also called atg8, is the major mammalian Atg and is divided into two types: LC3 I and LC3 II. As LC3 I is converted to LC3 II when suitable stimulation occurs, and the progression converts autophagosomes to autophagolysosomes, LC3 II is considered an autophagy marker, especially in macroautophagy $(15,16)$. In addition, many molecular proteins are involved in autophagy, such as PI3K and mTOR, and these proteins act as modulators in different stages of autophagy (17). However, little is known about the role of XPA in HCC or how it is affected by autophagy.

Studies on autophagy in HCC have become increasingly popular. Autophagy can be suppressed or activated in numerous malignancies by modulating the PI3K/Akt/ mTOR complex, and our previous study confirmed that IL37 could induce HCC cell autophagy via the PI3K/ Akt/mTOR pathway (18). In the last decade, evidence has suggested that Akt plays a central role in the PI3K/Akt/ mTOR signaling pathway and could predict sensitivity to antineoplastic therapies $(19,20)$. Most importantly, the PI3K/Akt/mTOR complex serves as an autophagy modulator by affecting antitumor activities $(21,22)$. However, there is no research about the modulation of $\mathrm{PI} 3 \mathrm{~K} / \mathrm{Akt} / \mathrm{mTOR}$ signaling in HCC by XPA.

In this study, we found that XPA was poorly expressed in HCC tissues and cells and XPA overexpression significantly suppressed HCC by inducing autophagy and apoptosis and inhibiting cell migration, invasion, and EMT in vitro. As all these biological functions seem to be associated with modulating the PI3K/Akt/mTOR pathway. Our study found a new mechanism for XPA inhibition of HCC via the $\mathrm{PI} 3 \mathrm{~K} / \mathrm{Akt} / \mathrm{m}$ TOR complex, XPA may be a novel therapeutic strategy for its treatment. We present the following article in accordance with the MDAR reporting checklist (available at https://dx.doi.org/10.21037/jgo-21-310).

\section{Methods}

\section{Regents and chemicals}

XPA overexpression lentivirus was purchased from Han Biotech (Hanbio, Shanghai, China) and the autophagy agonist rapamycin and autophagy inhibitor 3-MA were both purchased from MedChemExpress (MCE, NJ, USA). Recombinant human insulin-like growth factor 1 (IGF-1) was purchased from R \& D Systems (Minneapolis, Minnesota, USA). 


\section{Cell culture}

HL7702 cells are normal liver cells, and the HCC cell lines SMMC7721, Huh7, HepG2, Hep3B, SK-Hep-1, LM9, and Bel7402 were purchased from the American Type Culture Collection (ATCC, Manassas, VA, USA). High-sugar DMEM supplemented with $10 \%$ foetal bovine serum (FBS) (Gibco, California, USA) and 1\% penicillin/streptomycin (Beyotime, Shanghai, China) was used to culture the cells, which were incubated at $5 \% \mathrm{CO}_{2}$ and $37{ }^{\circ} \mathrm{C}$ (Thermo Forma; Thermo Fishier Scientific, Boston, USA).

\section{Stable overexpression XPA cell lines construction}

pHBLV-CMV-MCS-3FLAG-EF1-ZsGreen-T2Apuromycin (XPA) and hU6-MCS-Ubiquitin-stubRFPsenseGFP-LC3-IRES-puromycin (LC3) plasmids were purchased from Han Biotech (Hanbio, Shanghai, China) and Gene Chem Biotech (GeneChem, Shanghai, China). HEK293T cells were used to finish the construction and cotransfection procedure. The direct target sequence was synthesized and ligated into the vector, the supernatant was collected and then stored at $-80{ }^{\circ} \mathrm{C}$, and vectors expressing $\mathrm{XPA}, \mathrm{LC} 3$, and the empty vector were constructed by inserting the corresponding open reading frame (ORF) sequence. Hep3B and Huh7 cells were infected with lentivirus at multiplicities of infection (MOIs) ranging from 10-30 and $72 \mathrm{~h}$ later, cells stably expressing XPA were selected with $2 \mu \mathrm{g} / \mathrm{mL}$ puromycin.

\section{Terminal deoxynucleotidyl transferase dUTP nick end labelling (TUNEL) staining}

Hep3B and Huh7 cells infected with LV-NC and LVXPA were harvested then subjected to TUNEL staining to measure the apoptosis rate under confocal laser microscopy. The cells were stained with a YF ${ }^{\circledR 594}$ TUNEL Assay Apoptosis Detection Kit (US Everbright ${ }^{\circledR}$ Inc., California, USA).

\section{$R N A$ extraction and $R T-q P C R$ analysis}

TRIzol purchased from Takara (Takara, Dalian, China) was used to isolate the cell and tissue total RNA according to the manufacturer's protocols. All in one cDNA Synthesis Supermix $(5 \times)$ (Bimake Technology, Houston, TX, USA) was used to perform reverse transcription in a T100 Thermal Cycler machine (Bio-Rad, Berkeley,
CA, USA). $2 \times$ SYBR Green RT-qPCR master mix was also purchased from Bimake then subjected to PCR in the CFX 96 RT-qPCR reaction system (Bio-Rad, USA). Primers responsive to XPA were designed and synthesized by Sangon Biotech (Sangon Biotech, Shanghai, China). XPA-forward: 5'-CCAAAACAGAGGCAAAACAAGA-3'; XPA-reverse: 5'-GACTACCCCAAACTTCAAGAGA-3'; GAPDH-forward: 5'-CAACGTGTCAGTGGTGGA CCTG-3'; GAPDH-reverse: 5'-GTGTCGCTGTTGA AGTCAGAGGAG-3'. Amplification data were determined and are shown as $2^{-\Delta \Delta C_{t}}$.

\section{Immunobistochemistry (IHC)}

All procedures in this study involving human participants were in accordance with the Declaration of Helsinki (as revised in 2013). The study was approved by the ethics board of Yongchuan Hospital of Chongqing Medical University (No. 2020-98) and informed consent was taken from all the patients. Patient liver samples were fixed with formalin and embedded in paraffin for sectioning, and specific rabbit anti-XPA (1:100, Abways, Shanghai, China) was incubated with the sections at $4{ }^{\circ} \mathrm{C}$ overnight. After incubation with the primary antibody, an appropriate secondary antibody (ZSGB-BIO, Beijing, China) was used to incubate the sections at $37^{\circ} \mathrm{C}$ for $1 \mathrm{~h}$ and $\mathrm{DAB}$ (Boster, Wuhan, China) was used to activate the signal enhancement. All procedures were processed according to the manufacturers' instructions. Under high-power magnification, three images of each section were captured by ZEISS photograph system software (Carl ZEISS, Berlin, Germany).

\section{Confocal laser scanning microscopy}

Hep3B and Huh7 cells infected with LV-NC and LV$\mathrm{XPA}$ and with stable expression of LC3-GFP-mRFP were plated onto coverslips (Nest Biotechnology Co., Ltd., Jiangsu, China) for 24 h. Cells were then fixed with $4 \%$ paraformaldehyde for $15 \mathrm{~min}$ at room temperature and washed three times with PBS. Following this, the coverslips were soaked in DAPI solution for $15 \mathrm{~min}$ at room temperature then washed with PBS a further three times at 2 min per wash. Puncta of GFP and mRFP were calculated under confocal laser microscopy (Carl Zeiss, Berlin, Germany) by taking three random visual fields per coverslip. 


\section{Cell counting kit-8 assay}

The CCK-8 (Dojindo, Kumamoto, Japan) experiment was performed according to the manufacturer's instructions to determine the cell proliferation of Hep3B and Huh7 cells stably expressing XPA and their negative controls. Measure the absorbance at $450 \mathrm{~nm}$ with a microplate reader (Thermo, USA).

\section{Plate colony formation assay}

Hep3B and Huh7 cells stably expressing XPA and their negative controls were used to perform plate colony formation assay. Briefly, Hep3B and Huh7cells were suspended in High-sugar DMEM containing 10\% FBS and seeded in six-well culture plates at 1,000 cells per well. Triplicate cultures of each cell line were maintained at $37^{\circ} \mathrm{C}$ in a $5 \% \mathrm{CO}_{2}$ atmosphere, and fresh medium was fed every 7 days. After 2 weeks, colonies could be observed directly with the unaided eye. The colonies were fixed with $4 \%$ paraformaldehyde for $15 \mathrm{~min}$ at room temperature and stained with crystal violet for $15 \mathrm{~min}$ at room temperature. After washing 3 times with PBS (1x), the colonies plates were scaned by Panasonic scanner and then counted.

\section{Transwell wound-bealing assay}

Approximately $10^{5}$ Hep3B and Huh7 cells were suspended in the upper chamber of a 24-well Multiwell insert (BD) coated with 1:80 Matrigel solution to detect migration and invasion behavior. Complete culture media was then added to the lower chamber to attract the cells and $48 \mathrm{~h}$ later, Giemsa staining solution was used to stain the cells and the invading cells were counted under a microscope.

A wound-healing assay was performed by scraping the cultured cells with a $200-\mu \mathrm{L}$ pipette tip. The healing process was observed under a light microscope, and the images were captured randomly for up to three to five visuals. ImageJ software was used to measure the area of the scratches and calculate the width.

\section{Western blot analysis}

Total protein extraction was performed with RIPA lysis solution (Beyotime, Shanghai, China) and all samples were subjected to gel separation via SDS-PAGE (Beyotime, Shanghai, China). The proteins were then transferred to PVDF membranes (Merck Millipore, Ltd., Darmstadt,
Germany). QuickBlock ${ }^{\mathrm{TM}}$ (Beyotime, Shanghai, China) blocking buffer solution was used to block the PVDF membranes and TBST solution $(20 \mathrm{mM}$ Tris- $\mathrm{HCl}$, $137 \mathrm{mM} \mathrm{NaCl}$, and $0.1 \%$ Tween 20) was used to wash the membranes. Primary antibodies and their dilution ratio used in this study were as follows: rabbit anti-LC3B, rabbit anticleaved caspase-3, rabbit anti-Bax, rabbit anti-Bcl2, rabbit anti-p-PI3K (Try458), rabbit anti-PI3K, rabbit-p-AKT (Ser473), rabbit anti-AKT, rabbit anti-p-mTOR (Ser2448), and rabbit anti-mTOR antibodies were purchased from CST [1:1,000, Cell Signalling Technology, Inc., (CST), Danvers, MA, USA]; rabbit anti-Beclin1 $(1: 1,000)$, rabbit anti-p62 $(1: 1,000)$ and mouse anti-GAPDH $(1: 20,000)$ were purchased from Proteintech (Proteintech, Wuhan, China); and rabbit anti-XPA $(1: 1,000)$ was purchased from Abways (Abways, Shanghai, China). The primary antibodies were all incubated with the membranes at $4{ }^{\circ} \mathrm{C}$ overnight, after which, the membranes were washed with TBST before incubation with anti-rabbit immunoglobulin G (IgG) $(1: 20,000)$ (ZSGB-BIO; Beijing, China) and anti-mouse IgG (1:20,000) (ZSGB-BIO; Beijing, China) secondary antibodies for $1 \mathrm{~h}$ at room temperature. The washed membranes were then detected with an enhanced chemiluminescence (ECL) kit (Advansta, Menlo Park, CA, USA) and Vilber Fusion X7 Spectra (Fusion X7, France). Protein expression was measured by ImageJ software according to the manufacturer's instructions.

\section{Statistical analysis}

Data were expressed as the mean \pm standard deviation (SD) and student's $t$-test was used to compare the paired groups. One-way analysis of variance (ANOVA) followed by the Tukey-Kramer test for multiple comparisons was used to compare multiple groups in GraphPad Prism version 8 (GraphPad Software, La Jolla, CA, USA), and $\mathrm{P}<0.05$ was considered statistically significant.

\section{Results}

\section{XPA is poorly expressed in human HCC tissues and HCC cells}

To clarify the potential function of XPA in HCC, we first measured the expression of XPA in 48 paired adjacent non tumor liver tissues (ANLTs) and HCC tissues by Western blotting. Compared with the ANLTs, XPA expression was significantly lower in the HCC tissues, and the ratio was 

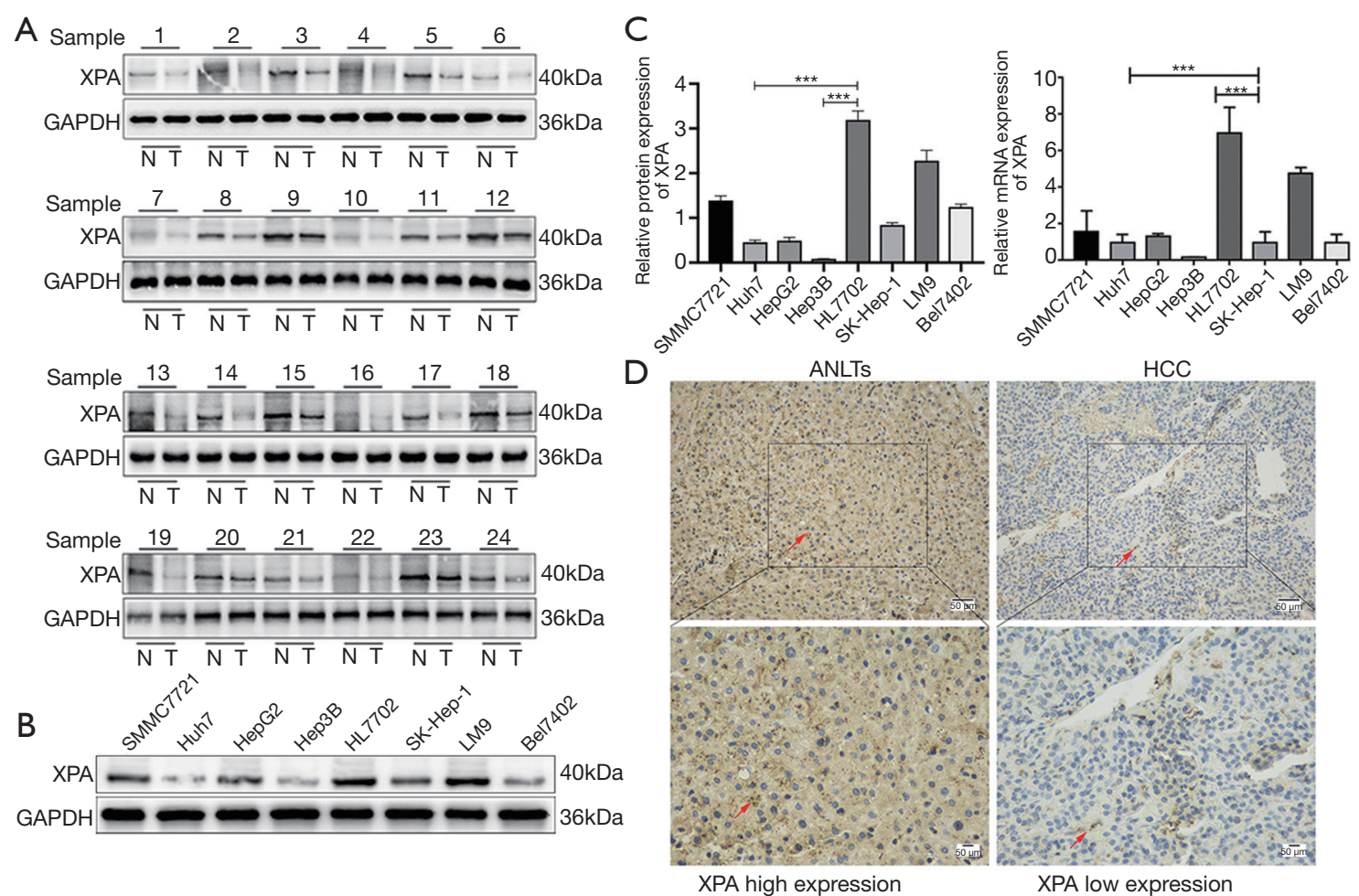

Figure 1 XPA is poorly expressed in HCC tissues and cells in vivo and in vitro. (A) Western blot analysis of XPA in human HCC tissues and ANLTs; (B,C) Western blot analysis of XPA in human normal and HCC cells and semiquantitative analysis of the data for each cell; (D) IHC detects XPA protein expression in human HCC tissues and ANLTs. The red arrow indicates XPA expression in hepatocytes. ***, P<0.001 vs. HL7702 cells, n=3/group. HCC, hepatocellular carcinoma; XPA, xeroderma pigmentosum group A; ANLTs, adjacent non tumor liver tissues.

almost $62.5 \%$ among all samples (Figure $1 A$ ). We then detected XPA expression in the HCC cell lines and normal hepatocytes by RT-qPCR and Western blot. Compared with nonneoplastic hepatocytes, Hep3B and Huh7 cells were the HCC cells with the lowest expression of SMMC7721, HepG2, SK-Hep-1, LM9, and Bel7402 at both the mRNA and protein levels (Figure 1B,1C). To confirm these results, we performed immunohistochemistry to analyze XPA expression in the HCC and ANLT tissues. Using previously described evaluation standards the intensity of IHC staining was scored according to the percentage of positive cells $(0,0 ;<5 \%, 1 ; 5-20 \%, 2 ; 20-60 \%, 3 ; 60-100 \%)$ and the magnitude of staining in the cells (low expression group: 0-1; high expression group: 2-3) (23). This showed XPA positive expression occurred mainly in the cytoplasm of the hepatocytes (Figure 1D), suggesting its expression is frequently low in human HCC, and that this may be a potential target for further investigation.

To obtain a better understanding of the co-relationship between XPA expression and the clinical manifestations of HCC, all 48 pairs of HCC and ANLT patients were divided into two groups: XPA high expression $(\mathrm{n}=30)$ and XPA low expression $(\mathrm{n}=18)$. This showed poor XPA expression was associated with age (Table 1), which indicated aged adults showed impaired XPA repair function.

\section{XPA overexpression significantly inbibits $\mathrm{Hep} 3 \mathrm{~B}$ and $\mathrm{Hub} 7$ cell proliferation in vitro}

Having confirmed that XPA is poorly expressed in HCC, to verify the molecular mechanism involved, we first evaluated the effect of XPA on the proliferation and growth ability of Hep3B and Huh7 cells in vitro. Lentivirus overexpressing XPA was introduced to infect Hep3B and Huh7 cells based on the results previously described and RT-qPCR and Western blotting were used to confirm the mRNA and protein expression of XPA. We found that XPA was significantly upregulated in both the LV-XPA groups of 
Table 1 Correlation between XPA expression and clinicopathological characteristics

\begin{tabular}{|c|c|c|c|}
\hline $\begin{array}{l}\text { Clinicopathological } \\
\text { characteristics }\end{array}$ & \multicolumn{2}{|c|}{ XPA protein expression } & $P$ value \\
\hline Age(years) & & & $<0.0001^{* * *}$ \\
\hline$<50$ & 11 & 17 & \\
\hline$\geq 50$ & 19 & 1 & \\
\hline Male & 24 & 16 & \\
\hline Female & 6 & 2 & \\
\hline Mass size $(\mathrm{cm})$ & & & 0.3512 \\
\hline$\leq 5$ & 11 & 4 & \\
\hline Absence & 6 & 5 & \\
\hline Presence & 24 & 13 & \\
\hline Histological differentiation & & & 0.0856 \\
\hline Poor & 3 & 0 & \\
\hline Moderate & 23 & 18 & \\
\hline Well & 4 & 0 & \\
\hline Serum AFP $(\mu \mathrm{g} / \mathrm{mL})$ & & & 0.7458 \\
\hline$>400$ & 8 & 6 & \\
\hline Absence & 10 & 2 & \\
\hline Presence & 20 & 16 & \\
\hline
\end{tabular}

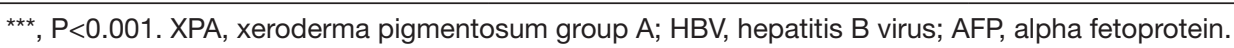

Hep3B and Huh7 cells compared with the LV-NC groups (Figure 2A-2C) and fluorescence microscopy showed strong zsGreen cells in the two cell lines (Figure 2D). In addition, colony formation was also inhibited in the LV-XPA groups compared with the LV-NC groups (Figure $2 E, 2 F$ ). Besides, XPA overexpression in could inhibit Hep3B and Huh7 cell proliferation in a time-dependent manner (Figure 2G,2H). These results indicate that XPA overexpression inhibits HCC by suppressing Hep3B and Huh7 cell proliferation and colony formation.

\section{Autophagy activation was induced by upregulating XPA in} $\mathrm{Hep} 3 \mathrm{~B}$ and $\mathrm{Hub} 7$ cells in vitro

Autophagy is a double-edged sword in many pathophysiologies, and our previous studies have confirmed that autophagy activation may have an HCC inhibitory function in vitro. Thus, we speculated that XPA overexpression may have the same biological function. To verify this hypothesis, we detected the expression of autophagy proteins by Western blot and found that LC3B II/I and Beclin1 protein 

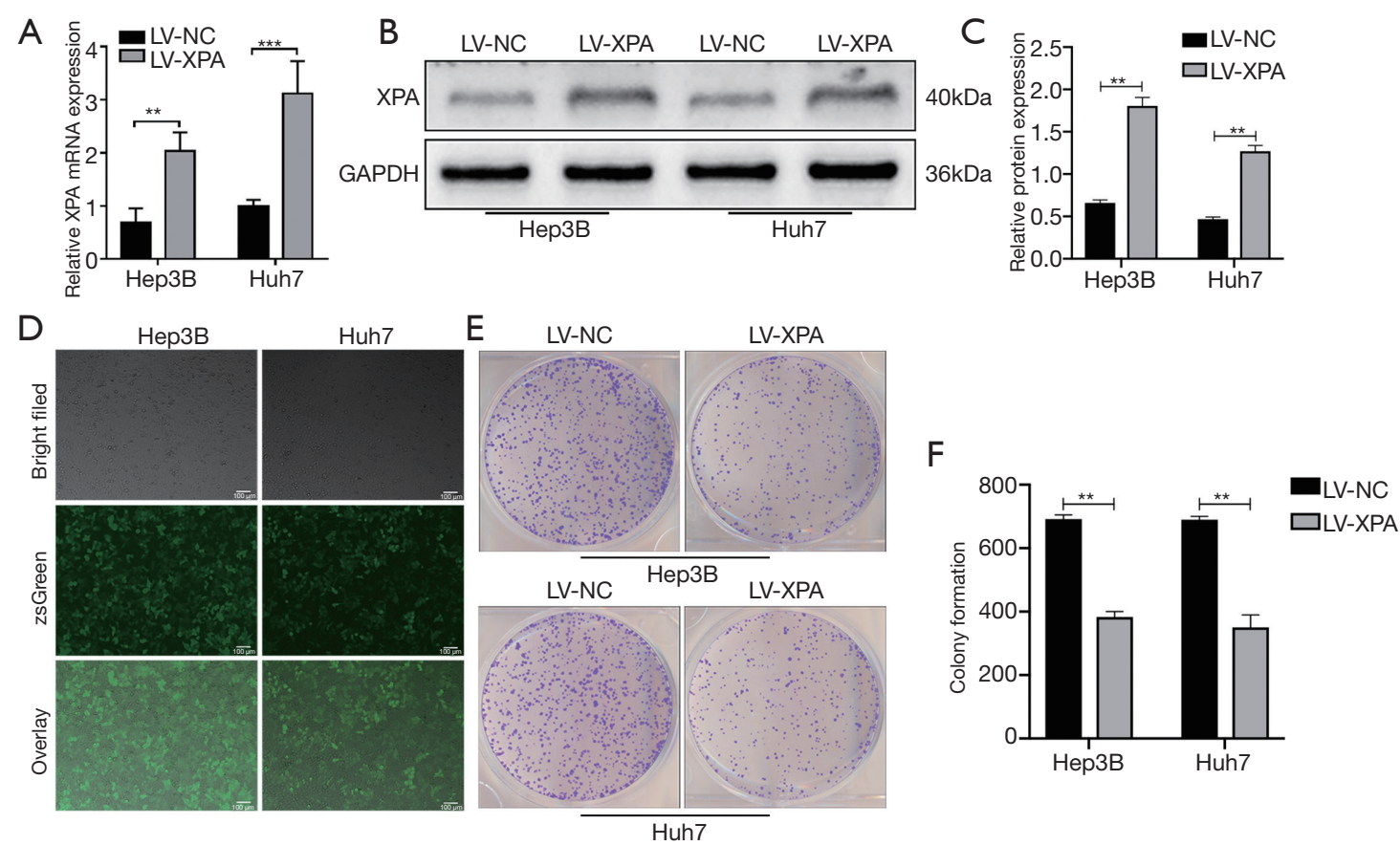

G
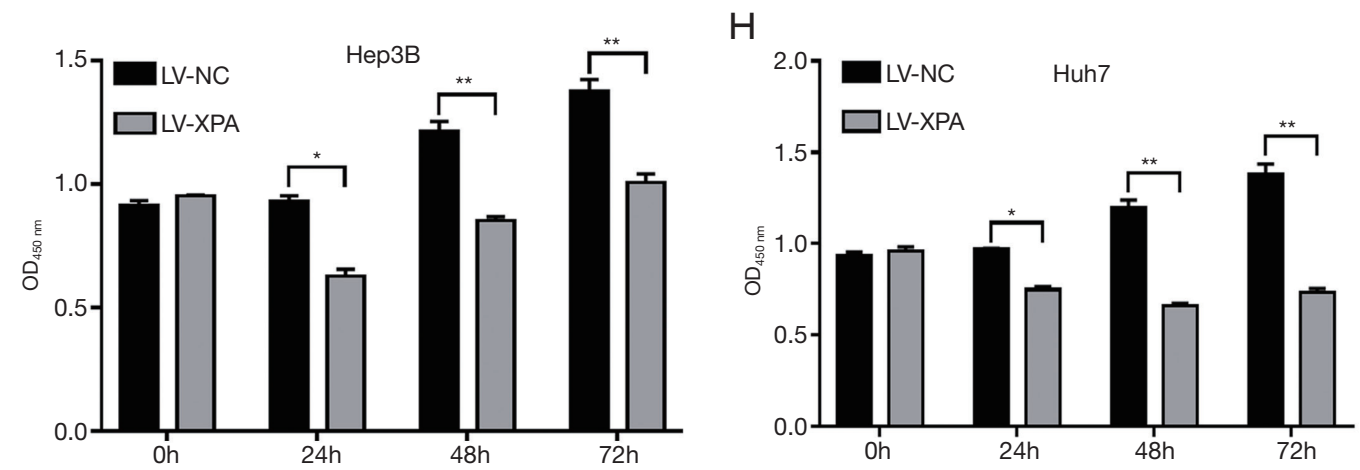

Figure 2 XPA inhibits HCC cell proliferation and colony formation. (A) The mRNA expression of XPA was detected by RT-qPCR analysis; $(\mathrm{B}, \mathrm{C})$ the protein expression of XPA was detected by Western blot analysis and semiquantitative analysis of the data for each group; (D) confocal laser microscopy reveals the lentivirus transfection efficiency in Hep3B and Huh7 cells; (E,F) colony formation assay showing Hep3B and Huh7 cell growth were stained by crystal violet staining solution (magnification: 100x); (G,H) cell proliferation was measured by performing a CCK-8 assay. ${ }^{*}, \mathrm{P}<0.05$; ${ }^{* *}, \mathrm{P}<0.01$; ${ }^{* *}, \mathrm{P}<0.001$ vs. LV-NC group, $\mathrm{n}=3$ /group. HCC, hepatocellular carcinoma; XPA, xeroderma pigmentosum group A; RT-qPCR, real time quantitative PCR.

expression were both much higher in the LV-XPA groups than in the LV-NC groups in Hep3B and Huh7 cells (Figure $3 A, 3 B$ ). As this study is mainly focused on autophagy, we introduced the LC3-GFP-mRFP lentivirus system to evaluate autophagic flux in vitro. GFP puncta reveal autophagosomes, and mRFP reveals autophagolysosomes. When the $\mathrm{pH}$ condition in cells changes towards acidity, autophagolysosomes will form, which means that the autophagic flux is smooth.
We applied confocal laser microscopy to detect puncta in Hep3B and Huh7 cells infected with lentivirus. As expected, upregulating XPA led to autophagosome (yellow puncta) formation in both Hep3B and Huh7 cells, and there were more autophagolysosome (red) puncta than GFP puncta, indicating the autophagic flux was partly smooth (Figure 3C,3D). Furthermore, we introduced rapamycin $(200 \mathrm{nM})$ and 3 -MA $(2 \mu \mathrm{M})(18)$ to prove that XPA overexpression induced autophagy in Hep3B and Huh7 cells. 

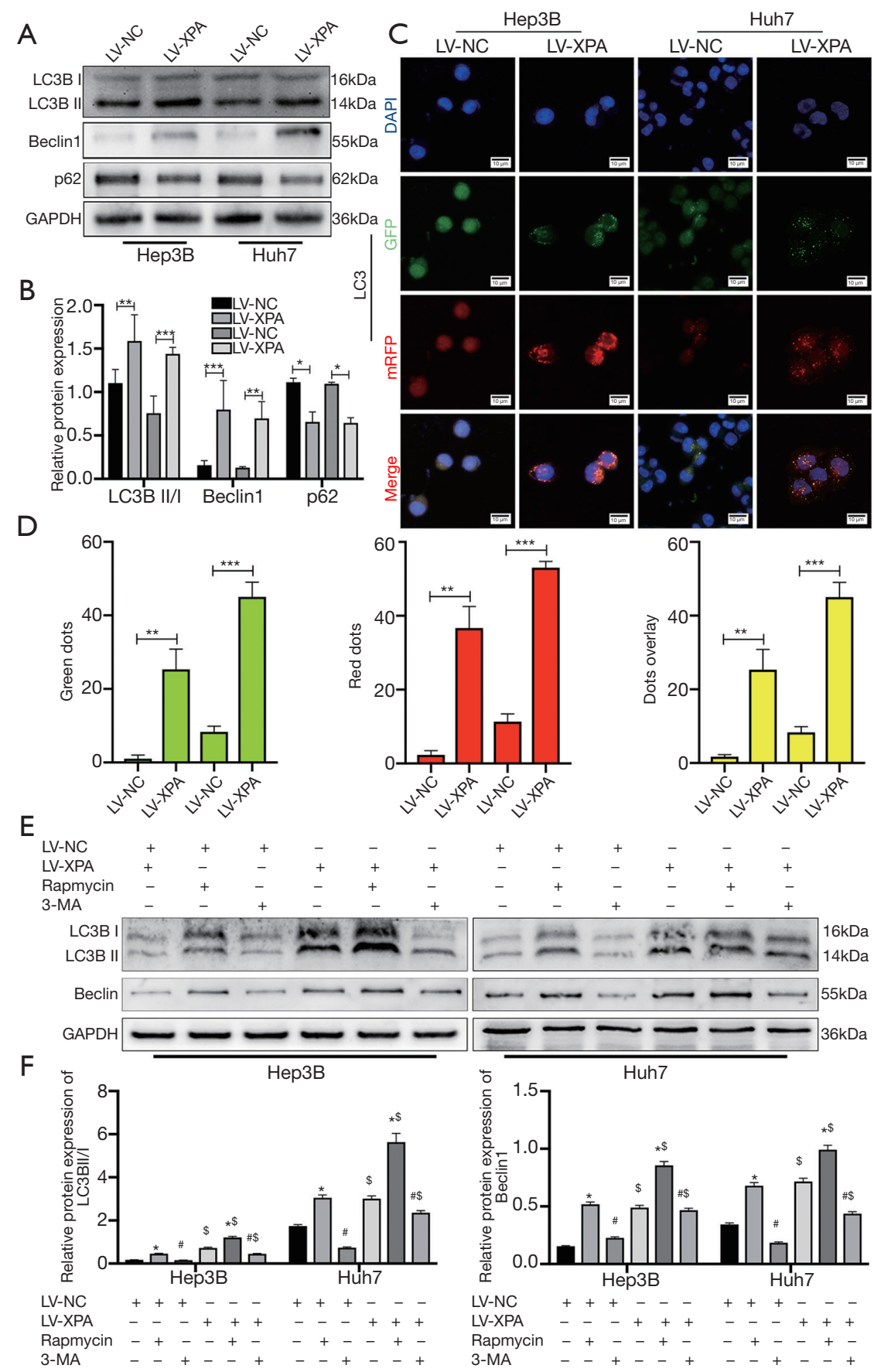

Figure 3 Autophagy activation was induced by upregulating XPA in Hep3B and Huh7 cells. (A,B) LC3B, Beclin1, and p62 protein expression was detected by Western blot analysis and semiquantitative analysis of data for each group in Hep3B and Huh7 cells. *, $\mathrm{P}<0.05$; **, $\mathrm{P}<0.01$; ***, $\mathrm{P}<0.001$ vs. LV-NC group, n=3/group. (C,D) Confocal laser scanning microscopy of Hep3B and Huh7 cells demonstrated the puncta number of GFP/RFP-LC3; colocalization of GFP and mRFP was visualized as yellow puncta (autophagosomes) in the LV-NC and LV-XPA groups. The red puncta represent autolysosomes, and the GFP puncta represent degradation depending on the acidity (scale bars: $10 \mu \mathrm{m}$ ), **, $\mathrm{P}<0.01$; ***, $\mathrm{P}<0.001$ vs. LV-NC group, n=3/group. (E,F) LC3B and Beclin1 protein expression was detected by Western blot analysis and semiquantitative analysis of data for each group after treatment with rapamycin $(200 \mathrm{~nm})$ and $3-\mathrm{MA}(2 \mu \mathrm{M}) .^{*}, \mathrm{P}<0.05$ vs. LV-NC group; \#, $\mathrm{P}<0.05$ vs. LV-NC group; ${ }^{\mathrm{S}}, \mathrm{P}<0.05$ vs. LV-NC group; ${ }^{*}, \mathrm{P}<0.05$ vs. LV-XPA group; and ${ }^{\# \$}, \mathrm{P}<0.05$ vs. LV-XPA group, $\mathrm{n}=3 /$ group. 

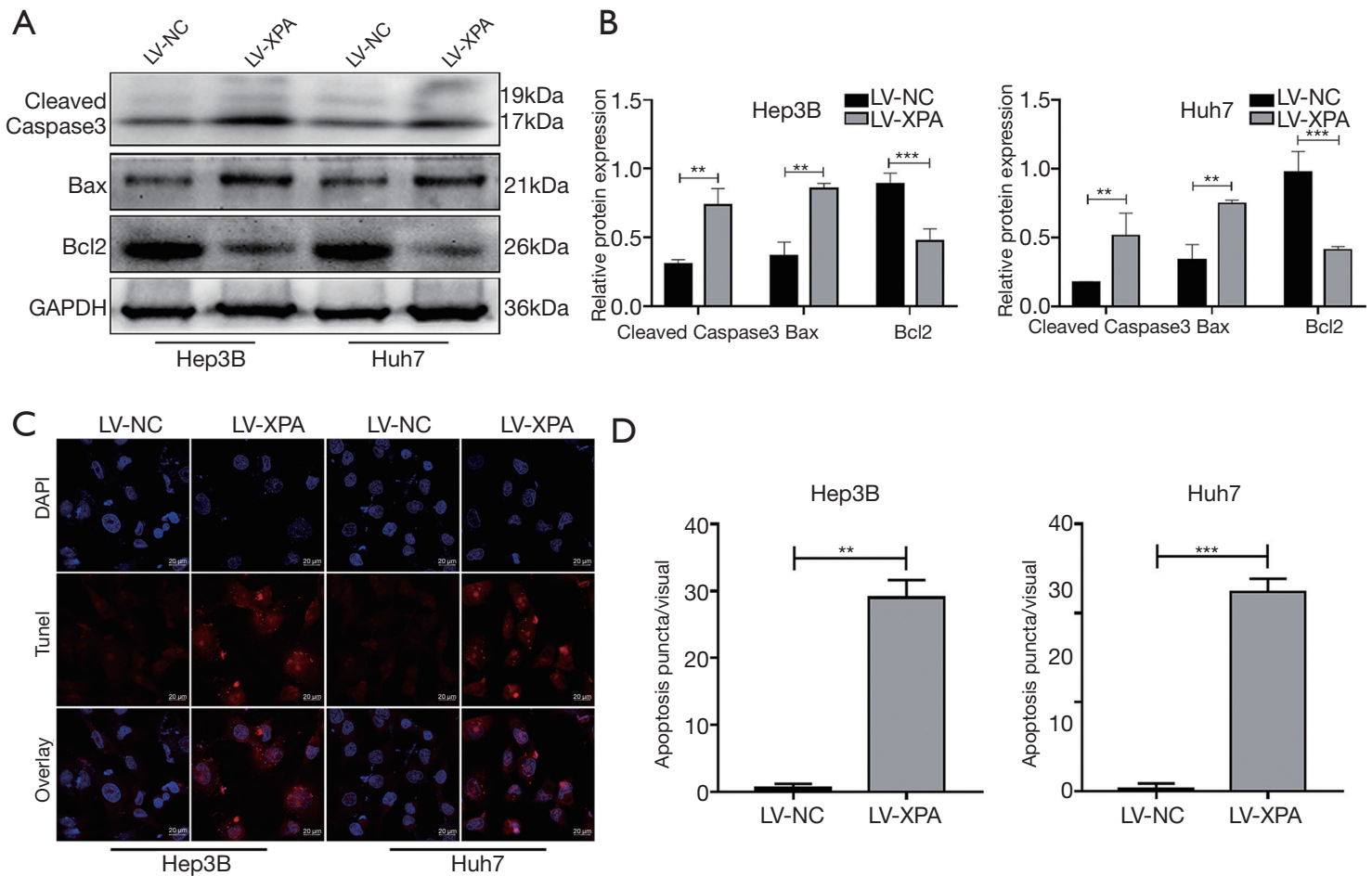

Figure 4 XPA overexpression triggers Hep3B and Huh7 cell apoptosis. (A,B) Cleaved-Caspase3, Bax, and Bcl2 protein expression was detected by Western blot analysis and semiquantitative analysis of the data for each group; (C,D) TUNEL staining revealed apoptotic puncta in Hep3B and Huh7 HCC cells and quantitative puncta number analysis $(400 \times)$. **, $\mathrm{P}<0.01 ;{ }^{* *}, \mathrm{P}<0.001$ vs. $\mathrm{LV}-\mathrm{NC}$ group, $\mathrm{n}=3$ / group. HCC, hepatocellular carcinoma.

The results showed that rapamycin and XPA both induced autophagy as reflected in higher LC3II/I and Beclin1 protein expression, and this function was much stronger when these two means were combined in the Hep3B and Huh7 cells. While 3-MA showed no autophagy inhibition function when used by itself, it suppressed XPA-induced autophagy activation, as reflected by a lower LC3II/I and Beclin1 protein expression level (Figure 3E, 3F). Taken together, upregulating XPA in Hep3B and Huh7 cells could significantly induce autophagy in vitro.

\section{XPA overexpression triggers $\mathrm{Hep} 3 \mathrm{~B}$ and $\mathrm{Hub} 7$ apoptosis in vitro}

Apoptosis and autophagy are two phenomena that often coexist in multiple conditions. The apoptosis gene $\mathrm{Bcl} 2$ is considered the connection between the autophagy gene Beclin 1 and apoptosis and interacts with the $\mathrm{BH} 3$ domain (24). Apoptotic proteins cleaved caspase-3, Bax, and
Bcl2 were detected by Western blotting and, as expected, the protein levels of cleaved caspase- 3 and Bax were increased in the LV-XPA groups, and Bcl2 was decreased compared with LV-NC groups in both Hep3B and Huh7 cells (Figure 4A,4B). Moreover, we performed TUNEL staining to confirm the Western blot results and showed the apoptotic puncta in the LV-XPA groups were much higher than those in the $\mathrm{LV}$-NC group in both Hep3B and Huh7 cells (Figure 4C,4D). These data indicate that XPA induces apoptosis in $\mathrm{HCC}$ in vitro.

\section{XPA overexpression inhibits migration, invasion, EMT, and wound bealing in vitro}

As E-cadherin, $\mathrm{N}$-cadherin, and vimentin are protein markers that reflect EMT in malignant tumors, we first detected their expression by Western blotting. This revealed E-cadherin protein expression was increased while $\mathrm{N}$-cadherin and vimentin expression were decreased in 


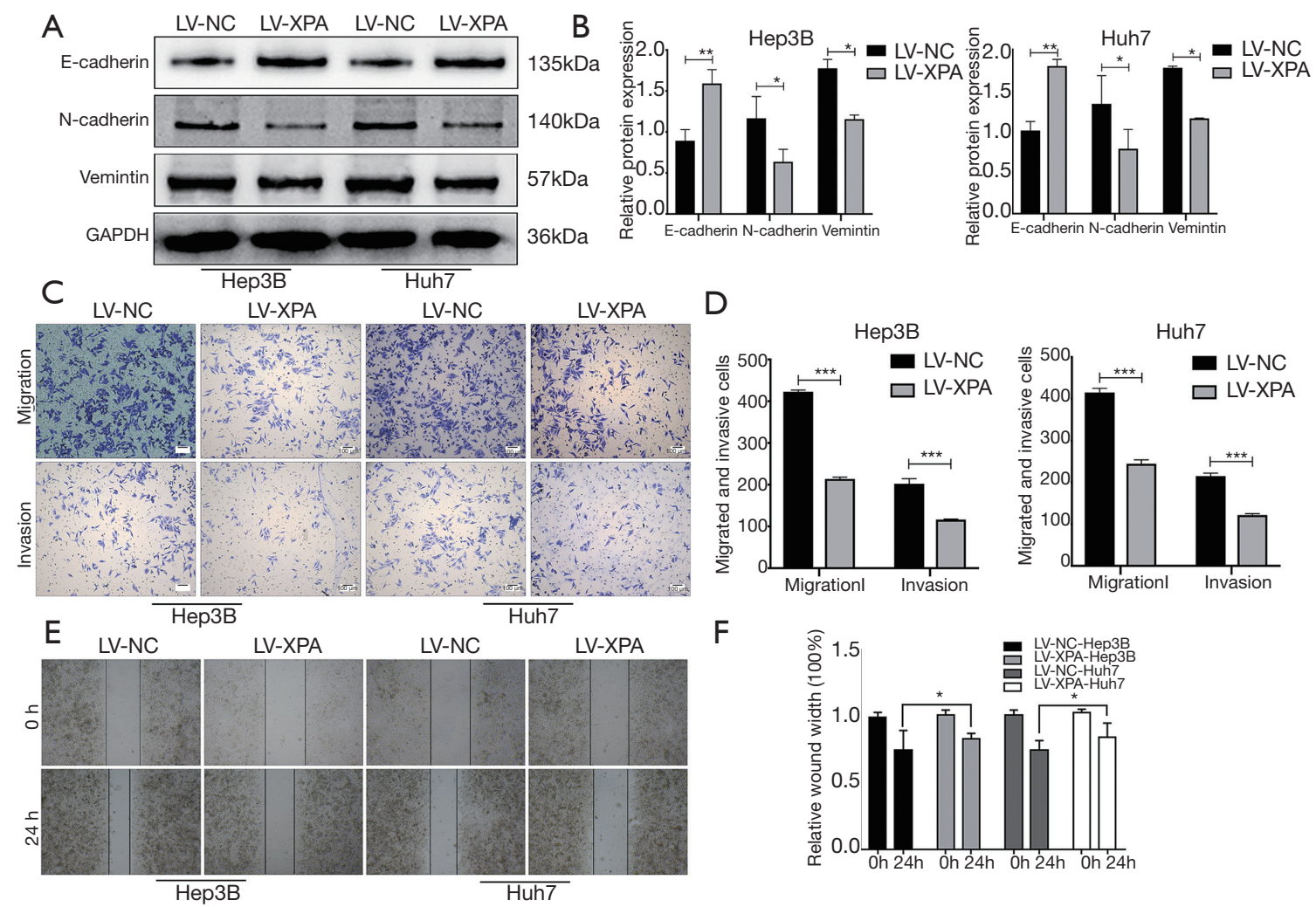

Figure 5 XPA overexpression inhibits migration, invasion, and EMT. (A,B) E-cadherin, N-cadherin, and vimentin protein expression was detected by Western blot and semiquantitative analysis of data for each group. (C,D) Representative images of the Transwell assay demonstrating Hep3B and Huh7 cell migration and invasion and the quantitative cell number of each group were stained by crystal violet solution. (E,F) Cell scratch test to evaluate the migration ability of Hep3B and Huh7 (magnification: $200 \times$ ). ${ }^{*}, \mathrm{P}<0.05$; ${ }^{* *}, \mathrm{P}<0.01$; and ***, $\mathrm{P}<0.001$ vs. LV-NC group, n=3/group. XPA, xeroderma pigmentosum group A; EMT, epithelial-mesenchymal transition.

the LV-XPA groups compared with the LV-NC groups in both the Hep3B and Huh7 cells (Figure 5A,5B). Cell migration and invasion behaviours are frequently used research objects to explore EMT in vitro, and in the present study, we applied a Transwell assay to detect cell migration and invasion in vitro. As expected, Hep3B and Huh7 cell migration and invasion were both significantly suppressed when cells were infected with lentivirus-XPA (Figure 5C,5D). In addition, by introducing the wound-healing assay, we then detected whether treatment with XPA overexpression in Hep3B and Huh7 cells would ameliorate the potential role of migration and invasion and found that a reduction in wound scratches could be seen in cells that overexpressed XPA after $24 \mathrm{~h}$ (Figure 5E, 5F). Taken together, these data indicate that XPA could suppress HCC migration, invasion, and EMT in vitro.

\section{XPA induces autophagy and suppresses HCC by modulating the PI3K/AKT/mTOR signaling complex}

There is evidence to support the hypothesis that the $\mathrm{PI} 3 \mathrm{~K} / \mathrm{AKT} / \mathrm{mTOR}$ signaling pathway is the classical autophagy molecular pathway and that it can be activated by IFG-1, which is known to be a tyrosine kinase activator (25). To obtain a better understanding of this molecular mechanism and whether PI3K/AKT/mTOR participates in XPA-induced autophagy activity, we first used different concentrations of IGF-1 to treat Hep3B and Huh7 cells and then detected p-AKT expression by Western blot. The results showed IGF-1 treatment led to the upregulation of p-AKT in an IGF-1 dose-dependent manner (Figure 6A-6D). We then combined XPA and IGF-1 to treat HCC cells to clarify the molecular mechanism and found LC3BII/I and 

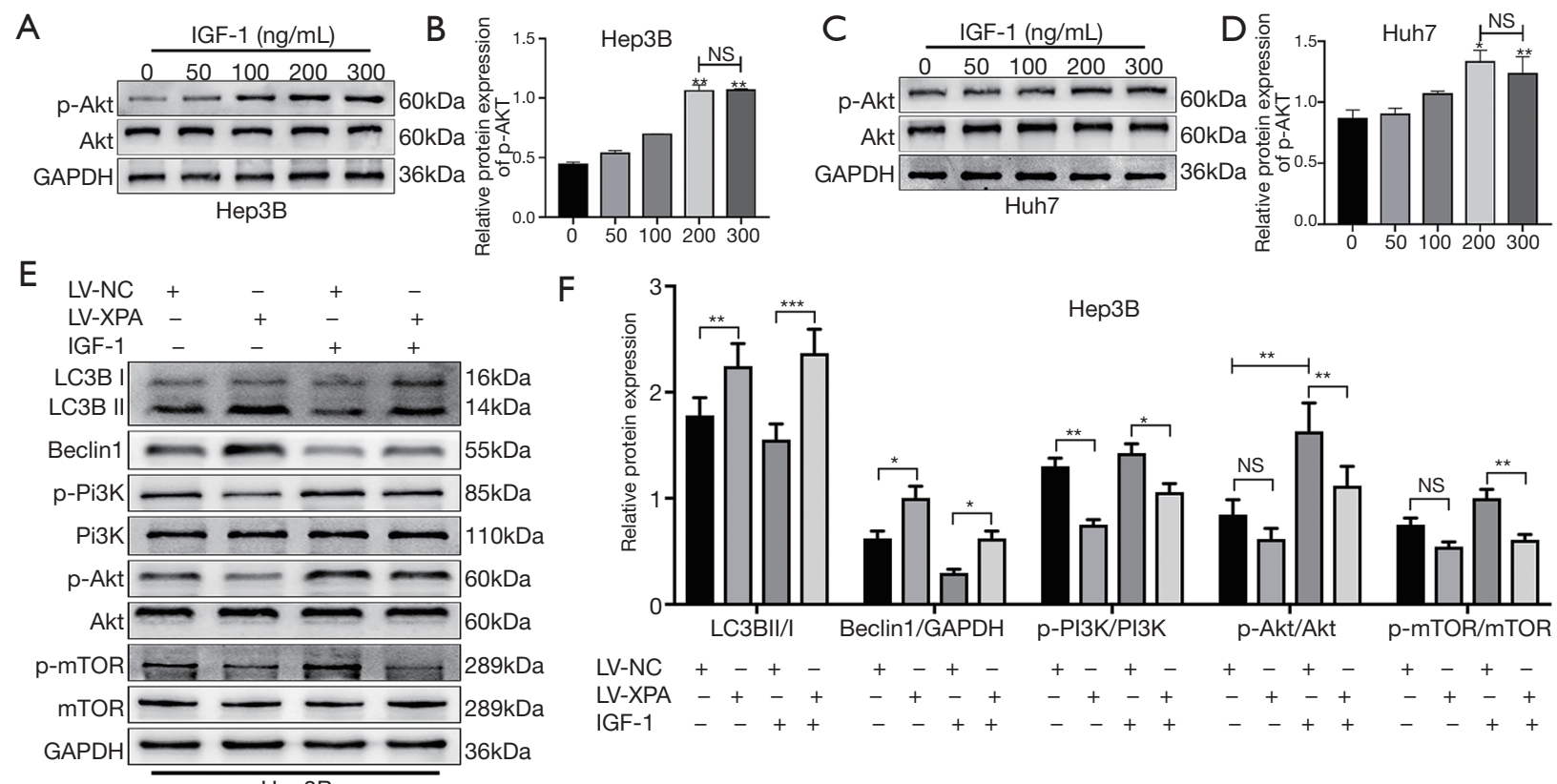

$\mathrm{F}$
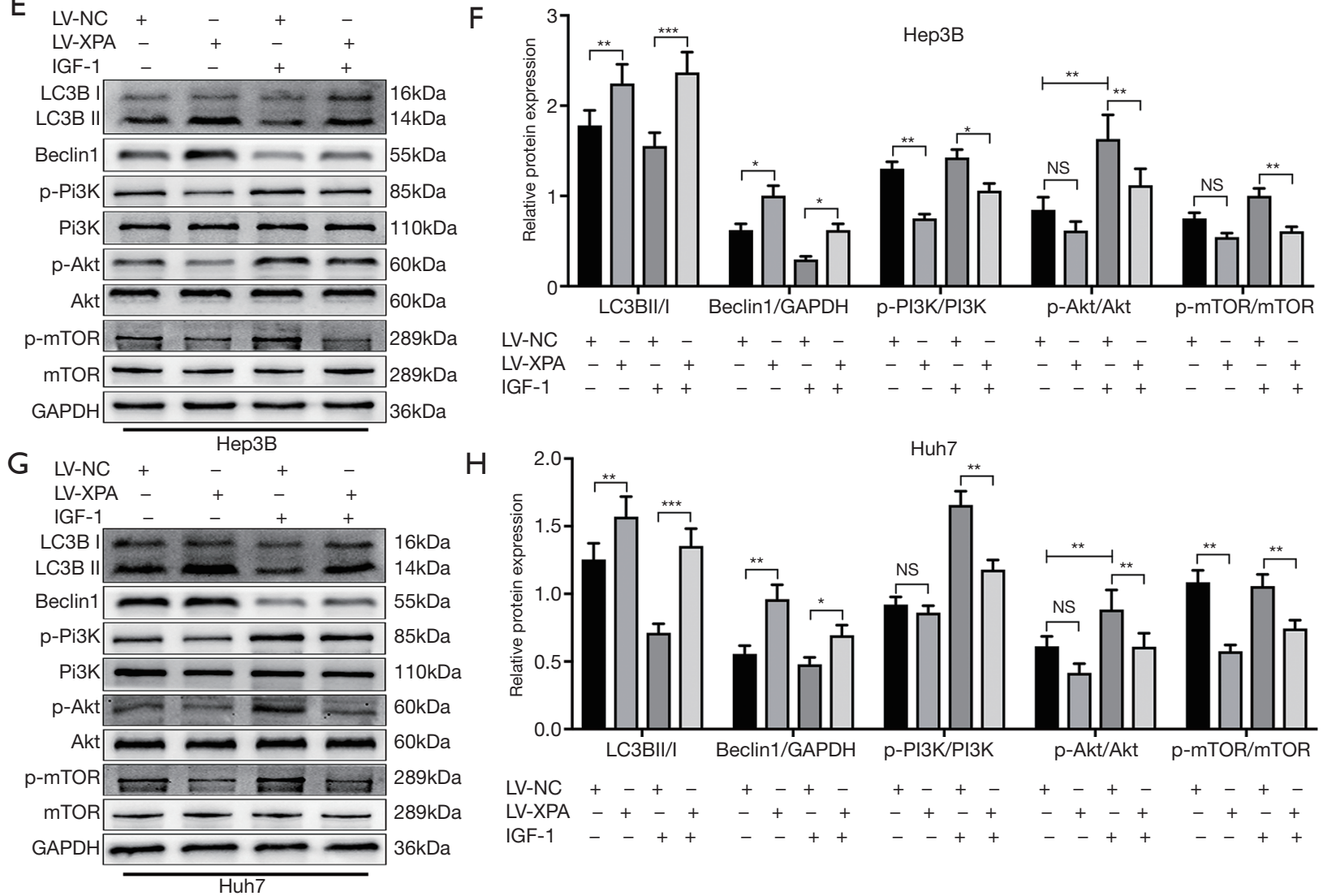

Figure 6 XPA induces autophagy and suppresses HCC by modulating the PI3K/AKT/mTOR signaling complex. (A-D) p-Akt and Akt protein expression was detected by Western blot and semiquantitative analysis of data for each group. ${ }^{*}, \mathrm{P}<0.05 ;{ }^{* *}, \mathrm{P}<0.01$; and ${ }^{* * *}, \mathrm{P}<0.001$ vs. LV-NC group, n=3/group. (E-H) LC3B, Beclin1, p-PI3K, PI3K, p-Akt, Akt, p-mTOR, and mTOR protein expression was detected by Western blot and semiquantitative analysis of data for each group in Hep3B and Huh7. HCC, hepatocellular carcinoma; XPA, xeroderma pigmentosum group A. NS: no significance.

Beclin1 were increased in LV-XPA, while IGF-1 significantly suppressed these protein levels. Further, total PI3K, $\mathrm{AKT}$, and mTOR protein levels were not altered, p-PI3K and $\mathrm{p}$-AKT protein levels were notably increased, while p-mTOR protein levels were decreased in both the Hep3B and Huh7 cells (Figure 6E-6H). These data indicate that autophagy activation by upregulating XPA was dependent on modulating the PI3K/AKT/mTOR signaling pathway.

\section{Discussion}

In this study we demonstrated that XPA was poorly expressed in HCC tissues and HCC cell lines, and XPA overexpression in vitro caused HCC suppression by modulating the PI3K/Akt/mTOR signaling pathway. This suggests triggering autophagy in HCC may be an effective therapeutic strategy for HCC management. To our knowledge, this is the first explanation of the biological 
function of XPA in inducing autophagy in HCC and additional efforts to elucidate the molecular mechanisms driving hepatic malignancies are urgently needed.

The poor expression of XPA has reported in numerous types of malignancies suggesting it could be used as a biomarker for prediction and intervention (26). Zhang et al. found that the XPA rs1800975 polymorphism could decrease the incidence and risk of breast cancer in a large sample of patients (27) and Prochnow et al. reported that high XPA expression significantly increased overall survival rates in patients with oropharyngeal squamous cell carcinomas (28). Further, Wada et al. revealed that XPA expression was decreased in uterine cervical cancer and could be a predictive marker of neoadjuvant chemotherapy (29). Despite these clinical findings, the detailed molecular mechanism by which XPA mediates cancer related responses and processes require elucidation. In line with the results of Prochnow et al., we found that XPA was poorly expressed in both HCC tissues and HCC cell lines and that its poor expression was associated with age, indicating aged adults have impaired XPA repair function. Unfortunately, we did not conduct a complete follow-up of patients, so no further analysis of the prognosis and survival rate was possible. Based on the results that XPA was downregulated in HCC tissues and HCC cell lines, we hypothesized that it might affect HCC proliferation in vitro. Colony formation assay and CCK- 8 assay experiments derived from the in vitro cell profiles confirmed that XPA overexpression significantly suppressed Hep3B and Huh7 cell proliferation and growth.

Autophagy plays an essential role in intracellular degradation by breaking down long-lived macromolecules such as cellular organelles and proteins to maintain intracellular homeostasis (30). Malignancies suffer from various stresses, and other stimuli may activate autophagy, producing a beneficial environment for malignant cells (31). Interestingly, Ge et al. demonstrated that XPA was significantly increased in cisplatin-resistant melanoma cells and that silencing XPA suppressed cisplatin-induced autophagy and induced cellular apoptosis by enhancing PARP1 (32). Given that XPA was poorly expressed in HCC tissues and HCC cell lines in this study, and we confirmed that XPA could suppress HCC cell proliferation and growth, we hypothesized that these effects might be due to it modulating autophagy.

To verify this hypothesis, we first tested the protein expression of the autophagy-related markers LC3BII/I, Beclin1, and p62, and found that LC3B II/I and Beclin1 were increased while p62 was decreased in both Hep3B and Huh7 cells. We then applied the LC3-GFP-mRFP lentivirus to detect autophagic flux in the cells, and the results were consistent with the Western blot analysis. To verify whether XPA truly inhibits HCC by inducing autophagy, we introduced the autophagy inducer rapamycin and the inhibitor 3-MA to verify its potential functions and, as expected, XPA induced HCC cell autophagy, and this effect was enhanced by rapamycin and reversed by 3-MA. Bcl2 and Beclin1 have been proven to be the core connection between autophagy and apoptosis in many pathophysiological processes (24) and in the present study, we performed Western blotting and TUNEL staining to confirm this hypothesis. As expected, XPA overexpression increased cleaved-Caspase-3, Bax, and Bcl2 protein expression. In addition, TUNEL staining showed a higher apoptosis rate in the LV-XPA groups than in the LV-NC groups in both Hep3B and Huh7 cells. Collectively, these data indicate that XPA serves as a regulator of autophagy and apoptosis in HCC, and these effects may be a novel therapeutic target for HCC.

The PI3K/Akt/mTOR signaling pathway can be activated by multiple stimulators, and the PI3K/Akt/ mTOR complex is the main cellular molecular pathway in autophagy $(33,34)$. IGF-1 is well known as the stimulator of the PI3K/Akt/mTOR pathway (25), and in this study, we first applied different concentrations of IGF-1 to activate p-Akt expression in both Hep3B and Huh7 cells. Here, we found that IGF-1 could activate p-Akt protein expression in a dose-dependent manner and the strongest effect was seen at $200 \mathrm{ng} / \mathrm{mL}$, so we chose this concentration to complete the following mechanistic research. We then combined XPA and IGF-1 to elucidate the molecular mechanism and the results showed that the protein expression of $\mathrm{p}-\mathrm{PI} 3 \mathrm{~K}$, p-Akt, and p-mTOR was decreased, while LC3B II/I and Beclin1 were increased with XPA treatment. These data indicate that XPA overexpression suppresses HCC and that this effect might occur through modulation of the PI3K/ Akt/mTOR signaling complex.

\section{Conclusions}

In summary, we have confirmed that XPA is poorly expressed in HCC and that XPA overexpression substantially suppressed HCC proliferation and growth by modulating the PI3K/Akt/mTOR signaling pathway. XPA could not only activate autophagy but also induce apoptosis in HCC. Therefore, this study provides a new biomarker and novel therapy method for the prediction and treatment of HCC 
which may have clinical applications. However, further animal and clinic research is needed for verification and development.

\section{Acknowledgments}

Funding: This work was supported by the National Natural Science Foundation of China (grant No. 81672959), the Joint Scientific Research Project of Chongqing Municipal Health and Health Committee and Chongqing Association for Science and Technology, Chongqing, China (grant No. 2018MSXM005), the 2021 Yongchuan District Science and Technology Project, Chongqing, China (grant No. 2021ycjckx20023); and the Postgraduate Innovation Project of Chongqing Education Commission, Chongqing, China (grant No. CYB19140).

\section{Footnote}

Reporting Checklist: The authors have completed the MDAR reporting checklist. Available at https://dx.doi. org/10.21037/jgo-21-310

Data Sharing Statement: Available at https://dx.doi. org/10.21037/jgo-21-310

Conflicts of Interest: All authors have completed the ICMJE uniform disclosure form (available at https://dx.doi. org/10.21037/jgo-21-310). The authors have no conflicts of interest to declare.

Ethical Statement: The authors are accountable for all aspects of the work in ensuring that questions related to the accuracy or integrity of any part of the work are appropriately investigated and resolved. This study was conducted in accordance with the Declaration of Helsinki (as revised in 2013) and was approved by the Ethics Committee of Yongchuan Hospital of Chongqing Medical University (No. 2020-98). Informed consent was taken from all the patients.

Open Access Statement: This is an Open Access article distributed in accordance with the Creative Commons Attribution-NonCommercial-NoDerivs 4.0 International License (CC BY-NC-ND 4.0), which permits the noncommercial replication and distribution of the article with the strict proviso that no changes or edits are made and the original work is properly cited (including links to both the formal publication through the relevant DOI and the license). See: https://creativecommons.org/licenses/by-nc-nd/4.0/.

\section{References}

1. Wu J. The changing epidemiology of hepatocellular carcinoma in Asia versus United States and Europe. Advances in Modern Oncology Research 2017;3.

2. Shiffman ML. The next wave of hepatitis $C$ virus: The epidemic of intravenous drug use. Liver Int 2018;38 Suppl 1:34-9.

3. Sugitani N, Sivley RM, Perry KE, et al. XPA: A key scaffold for human nucleotide excision repair. DNA Repair (Amst) 2016;44:123-35.

4. Camenisch U, Dip R, Schumacher SB, et al. Recognition of helical kinks by xeroderma pigmentosum group A protein triggers DNA excision repair. Nat Struct Mol Biol 2006;13:278-84.

5. Camenisch U, Nägeli H. XPA gene, its product and biological roles. Adv Exp Med Biol 2008;637:28-38.

6. Liu J, He C, Xing C, et al. Nucleotide excision repair related gene polymorphisms and genetic susceptibility, chemotherapeutic sensitivity and prognosis of gastric cancer. Mutat Res 2014;765:11-21.

7. Feng X, Liu J, Gong Y, et al. DNA repair protein XPA is differentially expressed in colorectal cancer and predicts better prognosis. Cancer Med 2018;7:2339-49.

8. Mei C, Hou M, Guo S, et al. Polymorphisms in DNA repair genes of XRCC1, XPA, XPC, XPD and associations with lung cancer risk in Chinese people. Thorac Cancer 2014;5:232-42.

9. Ding $\mathrm{D}$, Zhang $\mathrm{Y}, \mathrm{Yu} \mathrm{H}$, et al. Genetic variation of XPA gene and risk of cancer: a systematic review and pooled analysis. Int J Cancer 2012;131:488-96.

10. Zhi Y, Ji H, Pan J, et al. Downregulated XPA promotes carcinogenesis of bladder cancer via impairment of DNA repair. Tumour Biol 2017;39:1010428317691679.

11. Yang Z, Klionsky DJ. An overview of the molecular mechanism of autophagy. Curr Top Microbiol Immunol 2009;335:1-32.

12. Czaja MJ. Functions of autophagy in hepatic and pancreatic physiology and disease. Gastroenterology 2011;140:1895-908.

13. Yue Z, Jin S, Yang C, et al. Beclin 1, an autophagy gene essential for early embryonic development, is a haploinsufficient tumor suppressor. Proc Natl Acad Sci U S A 2003;100:15077-82.

14. Takamura A, Komatsu M, Hara T, et al. Autophagy- 
deficient mice develop multiple liver tumors. Genes Dev 2011;25:795-800.

15. Choi AM, Ryter SW, Levine B. Autophagy in human health and disease. N Engl J Med 2013;368:651-62.

16. Ogata M, Hino S, Saito A, et al. Autophagy is activated for cell survival after endoplasmic reticulum stress. Mol Cell Biol 2006;26:9220-31.

17. Lee YJ, Jang BK. The Role of Autophagy in Hepatocellular Carcinoma. Int J Mol Sci 2015;16:26629-43.

18. Li TT, Zhu D, Mou T, et al. IL-37 induces autophagy in hepatocellular carcinoma cells by inhibiting the PI3K/ AKT/mTOR pathway. Mol Immunol 2017;87:132-40.

19. Xu S, Li Y, Lu Y, et al. LZTS2 inhibits PI3K/AKT activation and radioresistance in nasopharyngeal carcinoma by interacting with p85. Cancer Lett 2018;420:38-48.

20. Park J, Feng J, Li Y, et al. DNA-dependent protein kinase-mediated phosphorylation of protein kinase $\mathrm{B}$ requires a specific recognition sequence in the $\mathrm{C}$-terminal hydrophobic motif. J Biol Chem 2009;284:6169-74.

21. Porta C, Paglino C, Mosca A. Targeting PI3K/Akt/mTOR Signaling in Cancer. Front Oncol 2014;4:64.

22. Wang SS, Chen YH, Chen N, et al. Hydrogen sulfide promotes autophagy of hepatocellular carcinoma cells through the PI3K/Akt/mTOR signaling pathway. Cell Death Dis 2017;8:e2688.

23. Levi L, Lobo G, Doud MK, et al. Genetic Ablation of the Fatty Acid-Binding Protein FABP5 Suppresses HER2Induced Mammary Tumorigenesis. Cancer Research 2013;73:4770-80.

24. Maiuri MC, Zalckvar E, Kimchi A, et al. Self-eating and self-killing: crosstalk between autophagy and apoptosis. Nat Rev Mol Cell Biol 2007;8:741-52.

25. Choucair A, Pham TH, Omarjee S, et al. The arginine

Cite this article as: Deng Y, Chen QS, Huang WF, Dai JW, Wu ZJ. XPA serves as an autophagy and apoptosis inducer by suppressing hepatocellular carcinoma in a PI3K/Akt/mTOR dependent manner. J Gastrointest Oncol 2021;12(4):1797-1810. doi: 10.21037/jgo-21-310 methyltransferase PRMT1 regulates IGF-1 signaling in breast cancer. Oncogene 2019;38:4015-27.

26. Borszéková Pulzová L, Ward TA, Chovanec M. XPA: DNA Repair Protein of Significant Clinical Importance. Int J Mol Sci 2020;21:2182.

27. Zhang Y, Guo Q, Yin X, et al. Association of XPA polymorphism with breast cancer risk: A meta-analysis. Medicine (Baltimore) 2018;97:e11276.

28. Prochnow S, Wilczak W, Bosch V, et al. ERCC1, XPF and XPA-locoregional differences and prognostic value of DNA repair protein expression in patients with head and neck squamous cell carcinoma. Clin Oral Investig 2019;23:3319-29.

29. Wada T, Fukuda T, Shimomura M, et al. XPA expression is a predictive marker of the effectiveness of neoadjuvant chemotherapy for locally advanced uterine cervical cancer. Oncol Lett 2018;15:3766-71.

30. Masiero E, Sandri M. Autophagy inhibition induces atrophy and myopathy in adult skeletal muscles. Autophagy 2010;6:307-9.

31. Eskelinen EL. The dual role of autophagy in cancer. Curr Opin Pharmacol 2011;11:294-300.

32. Ge R, Liu L, Dai W, et al. Xeroderma Pigmentosum Group A Promotes Autophagy to Facilitate Cisplatin Resistance in Melanoma Cells through the Activation of PARP1. J Invest Dermatol 2016;136:1219-28.

33. Huo R, Wang L, Liu P, et al. Cabazitaxel-induced autophagy via the PI3K/Akt/mTOR pathway contributes to A549 cell death. Mol Med Rep 2016;14:3013-20.

34. Mizushima N, Komatsu M. Autophagy: renovation of cells and tissues. Cell 2011;147:728-41.

(English Language Editors: B. Draper) 